\title{
Ultra-thin resin embedding method for scanning electron microscopy of individual cells on high and low aspect ratio 3D nanostructures
}

\author{
Andreea Belu ${ }^{1 \ddagger}$, Jan Schnitker ${ }^{1 \ddagger}$, Sergio Bertazzo ${ }^{2}$, Elmar Neumann ${ }^{1}$, Dirk Mayer ${ }^{1}$, Andreas \\ Offenhäusser $^{1}$, Francesca Santoro $^{1 \dagger *}$ \\ ${ }^{1}$ Institute of Complex Systems and Peter Grünberg Institute (ICS-8/PGI-8) - Bioelectronics, \\ Forschungszentrum Jülich GmbH, Jülich, Germany \\ ${ }^{2}$ Department of Medical Physics \& Biomedical Engineering, University College London, Malet \\ Place Engineering Building, London WC1E 6BT, UK.
}

*Corresponding author:

Francesca Santoro, Leo Brandt Straße 1, 52425, Jülich, Germany

E-mail: f.santoro@fz-juelich.de

$\dagger$ Current affiliation:

Department of Chemistry, Stanford University, Stanford, CA 94306, USA

E-mail: santorof@stanford.edu

$\$$ These authors have equal contributions 


\begin{abstract}
The preparation of biological cells for either scanning or transmission microscopy requires a complex process of fixation, dehydration and drying. Critical point drying (CPD) is commonly used for samples investigated with a scanning electron beam, while resin-infiltration is typically used for transmission electron microscopy. CPD may cause cracks at cellular surface and a sponge-like morphology of non-distinguishable intracellular compartments. Resin-infiltrated biological samples result in a solid block of resin, which can be further processed by mechanical sectioning. Here, we propose a method for removing resin excess on biological samples before effective polymerization. In this way the cells result to be embedded in an ultra-thin layer of epoxy resin. This novel method highlights in contrast to standard methods the imaging of individual cells not only on nanostructured planar surfaces but also on topological challenging substrates with high aspect ratio 3D features by SEM.
\end{abstract}

\title{
Keywords
}

Scanning electron microscopy, epoxy-based resins, cells, chemical fixation, ultrastructures.

\section{Introduction}

Understanding the interaction of cells with biomaterials has become a very broad field of research spanning over various methods of investigation and applications such as implants(Recum and Kooten, 1996), neuroimplants(Wang et al., 2005), biofilm formation(Pavithra and Doble, 2008), stem cell differentiation(Burdick and Vunjak-Novakovic, 2008), and can lead to e.g. cell differentiation, adhesion and affinity towards the matierial. Standard techniques such as fluorescence microscopy(Axelrod, 2001), surface plasmon microscopy(Toma et al., 2014) or electron microscopy(Friedmann et al., 2011; Santoro et al., 2014b; Wrobel et al., 2008) are powerful tools for the investigations of biological samples yet, but generally not allowing to achieve resolution in all three space dimensions at the nanoscale(Alivisatos et al., 2013).

It seems of foremost importance to establish an artifact-free preparation and visualization on the single cell level of any kind of surface. The imaging of cellular outgrowth with nanometer 
resolution with scanning electron microscopy is established since decades and recently allowed a full 3D volume reconstructions of cells and tissues(Bushby et al., 2011; Hoffpauir et al., 2007; Holzer and Cantoni, 2011; Villa et al., 2013). Nevertheless, traditional preparation techniques such as critical point drying (CPD) and air drying have some major limitations. Firstly, these two methods are notapplicable to every type of sample. Secondly, CPD and air drying induce typically volume shrinking artifacts in particular for confluent cells' monolayers(Schroeter et al., 1984) and, at last, only the sample surface is visible. For these reasons, alternative approaches have been carried out such as HDMS(Braet et al., 1997) biological sample preparation. salicylic acid. These methods are very efficient for SEM investigations of whole cells with volume preservation. In contrast, techniques such as thin resin plastification have been used for visualization of intracellular compartments with SEM(Bittermann et al., 2012; Kizilyaprak et al., 2014). Thin resin plastification method allows the preservation of intracellular structures but the draining of the resin is not sufficient to visualize the effective cell adhesion on the substrate. Cell interiors and the substrate-cell interaction is typically imaged by transmission electron microscopy or scanning electron microscopy, which requires cells to be embedded in a resin block and losing surface information, but gaining highly resolved access to cellular ultrastructures, in particular for slices of cells. This bulk like resin embedding favors a volume preservation of the cell avoiding structural and morphological artifacts due to the fact that the intracellular water component is basically replaced first with an intermediate liquid medium and, finally, with resin. In addition, mixtures of heavy metals-based compounds (i.e. osmium tetroxide, uranyl acetate) can be used to stain intracellular cell membranes and proteins prior to embedding, favoring contrast enhancement during the image acquisition. By using the additional technique of a focused ion beam, one is able to slice perpendicularly to the substrate surface through a sample and, achieving a tomographic imaging of the cell surface interface(Bushby et al., 2011; Drobne, 2013; Friedmann et al., 2011; Heymann et al., 2006; Knott et al., 2008; Lešer et al., 2009). A more complex scenario opens when cells interact with 3D nanoengineered substrates. Materials and geometries adopted during the nanostructures development can involve very challenging processes aimed to the investigation of $3 \mathrm{D}$ nanostructure-cell interaction and visualization of the actual interface. Many attempts have been carried out recently to characterize the tight adhesion between cell and 3D nano and microstructures with TEM and SEM with and 
without cross sectioning techniques (Angle et al., 2015; Bonde et al., 2014; Fendyur et al., 2011; Santoro et al., 2014a, 2014b; Spira and Hai, 2013).

Our approach tries to establish a novel embedding method, which brings the excellent properties of resin embedding of the TEM preparation to a single cell level by removing excess material and can be consequently imaged by a SEM and eventually prepared for FIB sectioning. This resin embedding approach of single cells becomes in particular relevant when high-aspect ratio spanning structures are analyzed and very thin and fragile cellular compartments need to be prevented from any damage.

(Duan et al., 2012; Hai et al., 2010; Lešer et al., 2009; Lin et al., 2014; Santoro et al., 2013; Spira et al., 2007; Tian et al., 2010; Wierzbicki et al., 2013; Xie et al., 2012, 2013).

Our new sample preparation procedure renders a determination of the exact contact area between surface and cell membrane possible.

\section{Material and methods}

\subsection{Cell culture}

\subsubsection{Substrates preparation}

The patterned samples were fabricated from a silicon wafer by depositing $100 \mathrm{~nm} \mathrm{Si}_{3} \mathrm{~N}_{4}$ and 100 $\mathrm{nm} \mathrm{SiO}_{2}$ by plasma-enhanced chemical vapor deposition (PECVD, Sentech, Germany). The pattern was generated by means of electron-beam lithography (EBPG 5000+ from Leica) followed by reactive ion etching (AMR RIE system from Oxford). The structures were etched to an overall depth of $100 \mathrm{~nm}$. The molds contained a pattern with lines and spaces with a width of $400 \mathrm{~nm}$ and a length of $4 \mu \mathrm{m}$.

All $\mathrm{Si}, \mathrm{Si} / \mathrm{SiO}_{2}$ (oxide thickness of $100 \mathrm{~nm}$ ), and $\mathrm{Si} / \mathrm{Si}_{3} \mathrm{~N}_{4} / \mathrm{SiO}_{2}$ substrates were cleaned with acetone for 15 minutes in a sonicator, 2-propanol (Merck, Darmstadt, Germany) and bi-distilled water in order to remove any possible organic residues from the substrate surface. Then, the samples were processed for surface activation in a plasma chamber (Plasma surface technology Pico, Diener electronic Company) using 1.4 mbar as pressure and a power of $200 \mathrm{~W}$ for 2 minutes. 3D gold mushroom-shaped microstructures were fabricated as shown in our former studies(Santoro et al., 2014a, 2014b). Afterwards, samples were prepared for cell culture, performing a sterilization under UV light (HS type, HERA Safe Company) for at least 30 
minutes. In addition, samples were coated with $10 \mathrm{mg} / \mathrm{ml}$ poly- $D$-lysine (PDL) diluted in Grey's Balance Salt Solution (GBSS, Life Technologies, Darmstadt, Germany) at $4^{\circ} \mathrm{C}$ overnight. Finally, samples were rinsed two times with GBSS shortly before cells' seeding.

\subsubsection{Neuronal culture}

Primary cortical neuronal cultures were prepared by removing cortices from Wistars rat embryos at embryonic day 18. Then, cortices were incubated with $0.25 \%$ trypsin/EDTA (Life Technologies) in a Petri dish (diameter of $35 \mathrm{~mm}$ ) for 5 minutes at $37^{\circ} \mathrm{C}$. After the incubation, the solution consisting of tissue and trypsin/ EDTA was transferred in to a $2 \mathrm{~mL}$ plastic tube. The tissue was let to settle at the bottom of the tube and the overlying trypsin/ EDTA was removed. Neurobasal ${ }^{\circledR}$ media (Life Technologies) was supplemented with 1\% (vol/vol) B27 (Invitrogen, Darmstadt, Germany), 0.25\% (vol/vol) L-glutamine (Invitrogen) and $0.1 \%$ (vol/vol) gentamycin antibiotic. One $\mathrm{mL}$ of supplemented Neurobasal ${ }^{\circledR}$ media at room temperature was added and, then, the tube was gently swirled by hand. This procedure was repeated 3 times. Finally, the supernatant was exchanged with $1 \mathrm{~mL}$ of fresh Neurobasal ${ }^{\circledR}$ media, and tissues were triturated until the cells were completely dissociated. A sample of cells re-suspension $(10 \mu \mathrm{L})$ was extracted, diluted in $20 \mu \mathrm{L}$ of supplemented Neurobasal ${ }^{\circledR}$ media and $10 \mu \mathrm{L}$ of trypan blue. This solution was placed in a cytometer (Neubauer chamber) for counting live and dead cells. After counting, the remaining cells were re-suspended in a final volume for being cultured on the substrates. The media was replaced completely 2 hours after seeding time. Every second day, half of the media was exchanged with freshly-prepared warm (supplemented) Neurobasal ${ }^{\circledR}$ media.

\subsubsection{HL-1 culture}

3D gold mushroom-shaped microstructures and control coverslips were washed and sterilized as mentioned earlier. Substrates were coated with fibronectin in 0.02\% Bacto TM gelatin (Fisher Scientific) for 1 hour, before cells' seeding. The HL-1 cells were cultured in a T-25 flask until confluency and dissociated with $1 \mathrm{~mL}$ of $0.25 \%$ trypsin/ EDTA for 5 minutes at $37^{\circ} \mathrm{C}$. The cells were re-suspended in $5 \mathrm{~mL}$ of Claycomb culture medium supplemented with $10 \%$ concentration ( $\mathrm{vol} / \mathrm{vol}$ ) of fetal bovine serum (Life Technologies), $100 \mu \mathrm{g} / \mathrm{mL}$ penicillin/streptomycin (Life Technologies), $0.1 \mathrm{mM}$ norepinephrine and $2 \mathrm{mM} \mathrm{L-glutamin}{ }^{35}$.The solution consisting of cells and media was transferred in a plastic $15 \mathrm{~mL}$ tube and centrifuged for 5 minutes at $1700 \mathrm{rpm}$. A 
cell pellet formed at the bottom of the tube. Supernatant liquid was removed and the cell pellet was carefully re-suspended by adding $1 \mathrm{~mL}$ of warm media pipetting up and down. $2 \%$ of cells at confluency was placed on each substrate and media was added to reach a final volume of 1.5 $\mathrm{mL}$. The cell culture media was replaced completely every day with fresh one.

\subsection{Sample preparation for scanning electron microscopy/focused ion beam}

After 4 DIV (days in vitro) neuronal cells and HL-1 cells were washed two times with warm phosphate buffer saline solution (PBS) and chemically fixed with a solution of $3.2 \%$ glutaraldehyde dissolved in PBS at pH 7.4 for 15 minutes at room temperature(Collins et al., 1977; Czeschik et al., 2015).Thereafter, samples were washed three times with PBS and, then, three times with bi-distillated water so as fixatives' residues could be completely removed. Gradually, water was replaced with an intermediate medium (ethanol) starting from a concentration of $10 \%$ up to $50 \%$ ( $\mathrm{vol} / \mathrm{vol})$. At this time, the incubation time was 5 minutes for each solution. Then, samples were infiltrated in sequence with a solution of $70 \%$ ethanol (vol/vol) for 15 minutes; $90 \% 95 \%$, and $100 \%$ (vol/vol) ethanol were exchanged three times and let for incubation for 5 minutes (each time). In the end, samples were stored in 100\% ethanol in a sealed dish at $4^{\circ} \mathrm{C}$.

Here, we present two different preparation methods of cells for SEM. The first method is CPD based on $\mathrm{CO}_{2}$ phase transition. The intermediate medium is exchanged to liquid $\mathrm{CO}_{2}$, and, by temperature increase, the samples is finally dried.

In particular, samples were transferred into the chamber of a critical point drying machine (CPD 030, BAL-TEC, Balzers, Liechtenstein) ensuring that the samples were continuously immersed in $100 \%$ ethanol. The system was slowly cooled down to $10^{\circ} \mathrm{C}$ (typically $1^{\circ} \mathrm{C} / \mathrm{step}$ ). The ethanol was gradually replaced with liquid $\mathrm{CO}_{2}$. This consist of typically 10 ethanol/ $\mathrm{CO}_{2}$ exchange repetitions. Then, the chamber's temperature was increased by $1{ }^{\circ} \mathrm{C} / \mathrm{step}$ until reaching a temperature of about $40^{\circ} \mathrm{C}$. At the critical temperature and pressure, the liquid $\mathrm{CO}_{2}$ turns into the supercritical state, the samples in the chamber completely dry off and, finally, the supercritical $\mathrm{CO}_{2}$ can be released via a control valve.

The second method consists of epoxy-based resin infiltration. First, we prepared the different components of the embedding polymer. $12.5 \mathrm{~mL}$ solution of Epon embedding medium was mixed solely with $20 \mathrm{~mL}$ dihydro-3-(tetrapropenyl)furan-2,5-dione (DDSA) for 5 minutes using a graduated plastic pipette (Fig. 1A). In parallel, a solution consisting of $17.5 \mathrm{~mL}$ Epon 
embedding medium and $15 \mathrm{~mL}$ methyl-5-norbornene-2,3-dicarboxylic anhydride (MNA) was prepared in a plastic tube as shown in Figure 1B. Afterwards, the two solutions were poured at the same time into a plastic container and $1.3 \mathrm{~mL}$ of 2,4,6-tris(dimethylaminomethyl)phenol (DMP30) was added. At this step, the color of the solution is typically red to orange (Fig. 1C). The solution is stirred for about 1 hour at room temperature until the solution's color turns into yellow, as exemplary shown in Figure 1D. Finally, the epoxy solution can be directly used for infiltration or, stored in plastic syringes at $-20^{\circ} \mathrm{C}$ and used on a later stage, after being thaw out for at least 10 minutes at room temperature.

The final embedding was carried out by replacing the $100 \%$ ethanol in which the samples were immersed, prepared as described earlier, with the yellow epoxy-based polymer. This procedure was gradually performed starting with a solution of ethanol/resin in a 3 to 1 proportion incubated for 3 hours at room temperature followed by two solutions in 2 to 1 and 1 to1 proportions incubated for 3 hours and overnight, respectively. Then, solutions with ethanol/resin proportions of 1 to 2 and 1 to 3 were incubated for 3 hours and, in the end, the samples were infiltrated only with pure epoxy resin (Fig. 1E). Considering that most of the chemical components are highly toxic, the complete embedding procedure was done in a hood under laminar flow conditions. The final step of our resin embedding method is the removal of excess viscous resin surrounding the sample (Fig. 1F). This is achieved by quickly splashing down ethanol on the back of the sample first and, afterwards, on the substrate's side where the cells adhered. As reference, we used a 5 $\mathrm{mL}$ plastic pipette to splash $5 \mathrm{~mL}$ of $100 \%$ ethanol at every repetition. The distance of the pipette from the substrate was about $1 \mathrm{~cm}$. We carried out experiments testing the effect of different numbers of repetition onto the final cell morphology. For this purpose, we performed splashing for $1,5,10,30$ times. The resin polymerization was achieved by heating the resin-embedded samples for 12 hours at $60^{\circ} \mathrm{C}$.

\subsection{Scanning electron microscopy imaging and focused ion beam sectioning}

The samples were coated with a thin layer of iridium (15-30 s deposition time, 15mA current) via sputtering deposition (K575X Sputter Coater, Quorum EMITECH, Ashford, UK). Then, each sample was mounted on a typical electron microscopy stub using a carbon adhesive tape or liquid silver paste. Samples were observed either with a single scanning electron beam (Magellan 400, FEI, Oregon, U.S.A and 1550VP, Zeiss, Oberkochen, Germany) or, complementarily, with an electron and ion beam in a dual beam machine (Helios 600i NanoLab Dual-beam, FEI, 
Oregon, U.S.A). Images were acquired using a detector for secondary electrons (SE) or backscattered electrons (BSE). The final beam acceleration was obtained by fixing a voltage between $3 \mathrm{kV}$ and $10 \mathrm{kV}$, and a current of $21 \mathrm{pA}$.

In addition, we performed a transversal cross sectioning and we fixed the milling parameters as presented in our former studies (Santoro et al., 2014b). Briefly, the target area was covered with a protective layer of platinum. The layer of platinum was deposited in two steps. During the first step, a $0.5 \mu \mathrm{m}$ thick layer of platinum was deposited via electron beam induce deposition (EBID) fixing the machine's stage at $0^{\circ}$. In addition, the sample was tilted to $52^{\circ}$ and $0.3-0.5 \mu \mathrm{m}$ of platinum were deposited via ion beam induced deposition (IBID). A first cross section was created by an ion beam milling procedure (at $30 \mathrm{kV}$ and $0.74 \mathrm{nA}$ ) fixing a milling depth of $1 \mu \mathrm{m}$ (nominally valid for silicon). Finally, fine polishing was performed at $30 \mathrm{kV}$ with a current of 80 pA. The SEM imaging of the resulting section was performed using an 'in-lens' detector for both SE and BSE.

\section{Results and discussion}

Our main purpose is the minimization of volume shrinkage artifacts during electron microscopy preparation protocol for individual cells adhering on planar and high aspect ratio 3Dnanostructures. In addition to preservation of cells' components, we aim to develop a reliable resin embedding method for investigating cells' spreading and adhesion on to the 3D nanostructures. In our investigation, we compare the effects of critical point drying and resin embedding preparations by SEM investigations.

After finding an optimal ultra-thin resin thickness for external structure preservation, we compared resin embedded neuronal cells to neuronal cells prepared via an optimal CPD procedure(Santoro et al., 2014b).

In Fig. 1G, we schematically represent the effect of the two tested methods, with particular attention to the deformation of the cell membrane domain attaching the culture substrate, such as a substrate with 3D features (i.e. cylindrical or mushroom-shaped 3D nanostructures). 


\subsection{Ultra-thin resin layer embedding procedure for single cells cultured on 3D nanostructures.}

In the first part of our study, we optimized a thin-layer resin embedding procedure in order to reduce shrinkage artifacts, which could alter the cells' structures. Our method mainly consists of the excess resin's removal before polymerization in contrast to standard procedures, where the resin is first polymerized and, then, mechanically removed. In order to achieve that, we splashed ethanol on the sample several times in quick sequential order, so that cells are still impregnated with liquid resin and no resin left overs remain on the substrate. The number of rinsing repetitions is a very critical parameter since high amount of ethanol could penetrate into the cell and introduce artifacts during the resin polymerization process. In order to find an optimal resin thickness, we quantified the effective resin's thickness as a function of the rinsing repetitions' number, (final ethanol rinsing volume) as shown in Fig. 2. Considering a nominal ethanol volume of $5 \mathrm{~mL}$ for each repetition, we tested final ethanol volumes of $5 \mathrm{~mL}, 25 \mathrm{~mL}, 50 \mathrm{~mL}$, and $150 \mathrm{~mL}$ on an overall substrate area of $1.2 \mathrm{~cm}^{2}$ using planar $\mathrm{Si} / \mathrm{SiO}_{2}$ substrates with primary cortical neurons.

In case of the lowest amount of ethanol (only one splashing repetition equals to an overall volume of $5 \mathrm{~mL}$ ), the layer of residual resin is relatively thick (about $1 \mu \mathrm{m}$ ) and it was not even possible to clearly identify the neuronal cell body perimeter and neurites while using a maximum acceleration voltage of $10 \mathrm{kV}$, a current of $21 \mathrm{pA}$ and a secondary electron detection.

In contrast, using a nominal ethanol volume of $25 \mathrm{~mL}$ the layer thickness of residual resin decrease to $500 \mathrm{~nm}$. Under these conditions, it was possible to clearly identify single cells on the substrate and, thus, several regions of interest for imaging. Finally, we found an optimal resin thickness of about $5 \mathrm{~nm}$ in correspondence of an ethanol volume of $50 \mathrm{~mL}$ (Fig. 2). In this case, small neurites, filopodia or neurites branching without cracks were clearly observed. In contrast to that, when $150 \mathrm{~mL}$ of ethanol were used for resin removal, neuronal cell membrane and neurites exhibited damages and typical cracks due to the quick evaporation of ethanol and a lack of resin embedding the cell. In several cases, we even observed a partial or total detachment of cells from the culture substrate.

In addition to SEM investigation, we performed FIB transversal sectioning to prove that our sectioning method, previously adopted for CPD-based cells(Santoro et al., 2014a, 2014b) is also suitable for thin-layer embedded cells. As shown in Fig. 2 (insets), the typical sectioning 
curtaining artifacts are not present, if cross sections are polished with a voltage of $30 \mathrm{kV}$ and a current of $80 \mathrm{pA}$.

\subsection{Effective comparison of critical point $v s$. ultra- thin resin layer embedding of single cells.}

In the second part of our study, we directly compared the effects of critical point drying and thinlayer resin embedding preparation while using planar culture substrates and high aspect ratio 3D nanostructures.

We investigated planar substrates with HL-1 cells and primary rat cortical neurons. For cells prepared with CPD, it is clearly observable that the cell membrane has a porous morphology and this porosity can cease to a consistent damage (Figs. 3A,C). In contrast, thin-layer resin embedding leads to reduced artifacts as shown in Figs. 3B,D. In this case, the cell membrane appears continuous and, furthermore, cell structures such as cell-cell attachment points (i.e. gap junctions) are perfectly preserved as shown for HL-1 cells (Fig. 3B). Similar conditions are present in thin-layer embedded primary neurons where cell body and neurites do not show any structural damage or cracks (Fig. 3D) in contrast to strongly-damaged CPD based samples (Fig. 3C).

Considering the efficiency of resin preparation in comparison to CPD on planar substrates, we investigated a more complex engineered substrate consisting of high and low aspect ratio 3D gold nanostructures (mushroom-shape) with cardiomyocyte-like HL-1 cells adhering on them. In our former studies, cylinder-like nanostructures induce lower wrapping states than 3D mushroom-like nanostructure corresponding also to higher membrane deformation in case of mushroom-like 3D nanostructures(Santoro et al., 2014a). Therefore, we investigate two extreme scenarios with respect to aspect ratio and dimensions for a direct comparison of CPD and thinlayer resin preparation methods. On the one hand, we considered the effect of the critical point drying procedure of HL-1 cells on 3D mushroom-shaped nanostructures with low aspect ratio (Figs. 4A,B). In this case, the CPD itself can induce high surface tension during the drying procedure while the cell membrane is under a relative high deformation condition due to the shape of the nanostructure. The cells membrane is extremely porous (Fig. 4B) and in some parts cracks are visible. On the other hand, we evaluated the efficiency of the ultrathin-layer resin embedding procedure (50 mL ethanol volume used for splashing) of HL-1 cells cultured on high aspect ratio 3D mushroom-like nanostructures (Figs. 4C, D). Here, the cell membrane is under 
higher deformation conditions and, thus, likely to result in a structural collapse while wrapping cylindrical nanostructures. The chances of a crack or complete collapse of free-standing cellular projections depend on the length and lateral dimensions of the cellular outgrowth. The defect rate increases in particular in cases of early stage engulfment processes of the 3D nanoelectrodes. Filopodia can span large distances in the micrometer regime from the mushroom cap to the surrounding sample surface and therefore collapse easily in case of CPD preparation. This affects largely affects the quality of the electron microscopy investigations and, eventually, the analysis of the cell membrane deformation as a response to the 3D nanostructures. Thin-layer resin embedded cells on high aspect ratio mushroom-like nanostructures appeared to be integer and forming a continuous assembly around the 3D nanostructures, as representative shown in Figs. 4,D. Here, 3D nanostructures wrapped at the edge of the cell form an extremely tended morphology left completely intact by the embedding procedure.

\subsection{Preliminary investigations of ultra-thin resin layer embedded cells on 3D nanostructures with focused ion beam sectioning.}

In our former studies we optimized a procedure for performing a transversal sectioning of CPDprepared single cells with FIB inducing almost no curtaining effects(Santoro et al., 2014a, 2014a). In those cases, cells attend to maintain a sponge-like morphology'(Santoro et al., 2014a, 2013). CPD is restrictively limited to the observation of cell parts in direct contact with the planar substrate and with 3D nanostructures. In particular for 3D nanostructures, the deformation induced to intracellular structures is not observable in CPD-prepared cells. In these preliminary investigations, we only focused on defining a suitable sectioning method of ultra-thin resin embedded cells with FIB. Considering that we did not include any heavy metals compounds in our preparation, intracellular structures were not visible. We created transversal sections of primary neurons on planar substrates (Fig. 5A), HL-1 cells on 3D mushroom-like nanostructures (Fig. 5B) and primary neurons on grooved-like nanostructures (Fig. 5C-D). We found that the resin embedding does not alter the effective cell attachment on the different types of substrates. Moreover, in some cases (Figs. 5A,C) we were able to visualize some intracellular structures without using any contrast enhancement agent. 


\section{Conclusions}

We optimized an embedding preparation procedure in order to have a SEM investigation of single cells or cell- cell interaction with an ultra thin-resin embedding and cells' structures preservation. Typically, resin blocks are commonly investigated by TEM rather than SEM after sectioning. This occurs because of the resin's thickness, which is often in the millimeter range. Our innovative finding grants the possibility to observe resin embedded individual cells on planar glass, silicon/silicon oxide substrates and 3D engineered substrates (low and high 3D mushroom-shaped nanostructures) directly with SEM. In particular, we have shown that the proposed resin infiltration procedure preserve the entire cellular volume as well as small cellular features (i.e. neurites, cell-cell attachment points). Moreover, preliminarily, we investigated the possibility to section ultra-thin resin embedded cells with FIB. For future experiments, it would be of great interest to perform sequential cross sectioning of resin-embedded individual cells aiming at a high resolution, artefact-free 3D reconstruction with contrast enhancement agents. In particular, additional staining with heavy metal ions can lead to an enhanced contrast and make different intracellular structures better observable.

Chemicals. Unless otherwise noted, all the chemical were purchased by Sigma Aldrich (Munich, Germany).

\section{References}

Alivisatos, A.P., Andrews, A.M., Boyden, E.S., Chun, M., Church, G.M., Deisseroth, K., Donoghue, J.P., Fraser, S.E., Lippincott-Schwartz, J., Looger, L.L., Masmanidis, S., McEuen, P.L., Nurmikko, A.V., Park, H., Peterka, D.S., Reid, C., Roukes, M.L., Scherer, A., Schnitzer, M., Sejnowski, T.J., Shepard, K.L., Tsao, D., Turrigiano, G., Weiss, P.S., Xu, C., Yuste, R., Zhuang, X., 2013. Nanotools for Neuroscience and Brain Activity Mapping. ACS Nano 7, 1850-1866. doi:10.1021/nn4012847

Angle, M.R., Cui, B., Melosh, N.A., 2015. Nanotechnology and neurophysiology. Curr. Opin. Neurobiol. 32, 132-140. doi:10.1016/j.conb.2015.03.014

Axelrod, D., 2001. Total Internal Reflection Fluorescence Microscopy in Cell Biology. Traffic 2, 764-774. doi:10.1034/j.1600-0854.2001.21104.x

Bittermann, A.G., Schaer, D., Mitsi, M, Vogel, V, Wepf, R, 2012. Thin layer plastification vs. block embedding: two alternative preparation strategies for 3D-imaging of cultured cells and biofilms by FIB/SEM. Presented at the European Microscopy Conference, Manchester.

Bonde, S., Buch-Månson, N., Rostgaard, K.R., Andersen, T.K., Berthing, T., Martinez, K.L., 2014. Exploring arrays of vertical one-dimensional nanostructures for cellular investigations. Nanotechnology 25 , 362001. doi:10.1088/0957-4484/25/36/362001 
Braet, F., De Zanger, R., Wisse, E., 1997. Drying cells for SEM, AFM and TEM by hexamethyldisilazane: a study on hepatic endothelial cells. J. Microsc. 186, 84-87. doi:10.1046/j.1365-

2818.1997.1940755.x

Burdick, J.A., Vunjak-Novakovic, G., 2008. Engineered Microenvironments for Controlled Stem Cell Differentiation. Tissue Eng. Part A 15, 205-219. doi:10.1089/ten.tea.2008.0131

Bushby, A.J., P'ng, K.M.Y., Young, R.D., Pinali, C., Knupp, C., Quantock, A.J., 2011. Imaging threedimensional tissue architectures by focused ion beam scanning electron microscopy. Nat. Protoc. 6, 845-858. doi:10.1038/nprot.2011.332

Collins, V.P., Arborgh, B., Brunk, U., 1977. A Comparison of the Effects of Three Widely Used Glutaraldehyde Fixatives on Cellular Volume and Structure. Acta Pathol. Microbiol. Scand. [A] 85A, 157-168. doi:10.1111/j.1699-0463.1977.tb00413.x

Czeschik, A., Rinklin, P., Derra, U., Ullmann, S., Holik, P., Steltenkamp, S., Offenhäusser, A., Wolfrum, B., 2015. Nanostructured cavity devices for extracellular stimulation of HL-1 cells. Nanoscale 7, 9275-9281. doi:10.1039/C5NR01690H

Drobne, D., 2013. 3D Imaging of Cells and Tissues by Focused Ion Beam/Scanning Electron Microscopy (FIB/SEM), in: Sousa, A.A., Kruhlak, M.J. (Eds.), Nanoimaging, Methods in Molecular Biology. Humana Press, pp. 275-292.

Duan, X., Gao, R., Xie, P., Cohen-Karni, T., Qing, Q., Choe, H.S., Tian, B., Jiang, X., Lieber, C.M., 2012. Intracellular recordings of action potentials by an extracellular nanoscale field-effect transistor. Nat. Nanotechnol. 7, 174-179. doi:10.1038/NNANO.2011.223

Fendyur, A., Mazurski, N., Shappir, J., Spira, M.E., 2011. Formation of Essential Ultrastructural Interface between Cultured Hippocampal Cells and Gold Mushroom-Shaped MEA- Toward "IN-CELL" Recordings from Vertebrate Neurons. Front. Neuroengineering 4. doi:10.3389/fneng.2011.00014

Friedmann, A., Hoess, A., Cismak, A., Heilmann, A., 2011. Investigation of cell-substrate interactions by focused ion beam preparation and scanning electron microscopy. Acta Biomater. 7, 2499-2507. doi:10.1016/j.actbio.2011.02.024

Hai, A., Shappir, J., Spira, M.E., 2010. In-cell recordings by extracellular microelectrodes. Nat. Methods 7, 200-202. doi:10.1038/nmeth.1420

Heymann, J.A.W., Hayles, M., Gestmann, I., Giannuzzi, L.A., Lich, B., Subramaniam, S., 2006. Site-specific 3D imaging of cells and tissues with a dual beam microscope. J. Struct. Biol. 155, 63-73. doi:10.1016/j.jsb.2006.03.006

Hoffpauir, B.K., Pope, B.A., Spirou, G.A., 2007. Serial sectioning and electron microscopy of large tissue volumes for 3D analysis and reconstruction: a case study of the calyx of Held. Nat. Protoc. 2, 922. doi:10.1038/nprot.2007.9

Holzer, L., Cantoni, M., 2011. Review of FIB-tomography. Nanofabrication Using Focus. Ion Electron Beams Princ. Appl. 559201222.

Kizilyaprak, C., Daraspe, J., Humbel, B. m., 2014. Focused ion beam scanning electron microscopy in biology. J. Microsc. 254, 109-114. doi:10.1111/jmi.12127

Knott, G., Marchman, H., Wall, D., Lich, B., 2008. Serial Section Scanning Electron Microscopy of Adult Brain Tissue Using Focused Ion Beam Milling. J. Neurosci. 28, 2959-2964. doi:10.1523/JNEUROSCI.3189-07.2008

Lešer, V., Drobne, D., Pipan, Ž., Milani, M., Tatti, F., 2009. Comparison of different preparation methods of biological samples for FIB milling and SEM investigation. J. Microsc. 233, 309-319. doi:10.1111/j.1365-2818.2009.03121.x

Lin, Z.C., Xie, C., Osakada, Y., Cui, Y., Cui, B., 2014. Iridium oxide nanotube electrodes for sensitive and prolonged intracellular measurement of action potentials. Nat. Commun. 5. doi:10.1038/ncomms4206 
Pavithra, D., Doble, M., 2008. Biofilm formation, bacterial adhesion and host response on polymeric implants-issues and prevention. Biomed. Mater. 3, 034003. doi:10.1088/1748-6041/3/3/034003

Recum, A.F.V., Kooten, T.G.V., 1996. The influence of micro-topography on cellular response and the implications for silicone implants. J. Biomater. Sci. Polym. Ed. 7, 181-198. doi:10.1163/156856295X00698

Santoro, F., Dasgupta, S., Schnitker, J., Auth, T., Neumann, E., Panaitov, G., Gompper, G., Offenhäusser, A., 2014a. Interfacing Electrogenic Cells with 3D Nanoelectrodes: Position, Shape, and Size Matter. ACS Nano 8, 6713-6723. doi:10.1021/nn500393p

Santoro, F., Neumann, E., Panaitov, G., Offenhäusser, A., 2014b. FIB section of cell-electrode interface: An approach for reducing curtaining effects. Microelectron. Eng. 124, 17-21. doi:10.1016/j.mee.2014.04.021

Santoro, F., Schnitker, J., Panaitov, G., Offenhäusser, A., 2013. On Chip Guidance and Recording of Cardiomyocytes with 3D Mushroom-Shaped Electrodes. Nano Lett. 13, 5379-5384. doi:10.1021/nl402901y

Schroeter, D., Spiess, E., Paweletz, N., Benke, R., 1984. A procedure for rupture-free preparation of confluently grown monolayer cells for scanning electron microscopy. J. Electron Microsc. Tech. 1, 219-225. doi:10.1002/jemt.1060010302

Spira, M.E., Hai, A., 2013. Multi-electrode array technologies for neuroscience and cardiology. Nat. Nanotechnol. 8, 83-94. doi:10.1038/nnano.2012.265

Spira, M.E., Kamber, D., Dormann, A., Cohen, A., Bartic, C., Borghs, G., Langedijk, J.P.M., Yitzchaik, S., Shabthai, K., Shappir, J., 2007. Improved Neuronal Adhesion to the Surface of Electronic Device by Engulfment of Protruding Micro-Nails Fabricated on the Chip Surface, in: Solid-State Sensors, Actuators and Microsystems Conference, 2007. TRANSDUCERS 2007. International. Presented at the Solid-State Sensors, Actuators and Microsystems Conference, 2007. TRANSDUCERS 2007. International, pp. 1247-1250. doi:10.1109/SENSOR.2007.4300363

Tian, B., Cohen-Karni, T., Qing, Q., Duan, X., Xie, P., Lieber, C.M., 2010. Three-Dimensional, Flexible Nanoscale Field-Effect Transistors as Localized Bioprobes. Science 329, 830-834. doi:10.1126/science.1192033

Toma, K., Kano, H., Offenhäusser, A., 2014. Label-Free Measurement of Cell-Electrode Cleft Gap Distance with High Spatial Resolution Surface Plasmon Microscopy. ACS Nano 8, 12612-12619. doi:10.1021/nn505521e

Villa, E., Schaffer, M., Plitzko, J.M., Baumeister, W., 2013. Opening windows into the cell: focused-ionbeam milling for cryo-electron tomography. Curr. Opin. Struct. Biol., Protein-carbohydrate interactions / Biophysical methods 23, 771-777. doi:10.1016/j.sbi.2013.08.006

Wang, P., Xu, G., Qin, L., Xu, Y., Li, Y., Li, R., 2005. Cell-based biosensors and its application in biomedicine. Sens. Actuators B Chem., Proceedings of the Tenth International Meeting on Chemical Sensors IMCS - 102004 108, 576-584. doi:10.1016/j.snb.2004.11.056

Wierzbicki, R., Købler, C., Jensen, M.R.B., Łopacińska, J., Schmidt, M.S., Skolimowski, M., Abeille, F., Qvortrup, K., Mølhave, K., 2013. Mapping the Complex Morphology of Cell Interactions with Nanowire Substrates Using FIB-SEM. PLoS ONE 8, e53307. doi:10.1371/journal.pone.0053307

Wrobel, G., Höller, M., Ingebrandt, S., Dieluweit, S., Sommerhage, F., Bochem, H.P., Offenhäusser, A., 2008. Transmission electron microscopy study of the cell-sensor interface. J. R. Soc. Interface 5, 213-222. doi:10.1098/rsif.2007.1094

Xie, C., Lin, Z., Hanson, L., Cui, Y., Cui, B., 2012. Intracellular recording of action potentials by nanopillar electroporation. Nat. Nanotechnol. 7, 185-190. doi:10.1038/nnano.2012.8

Xie, X., Xu, A.M., Leal-Ortiz, S., Cao, Y., Garner, C.C., Melosh, N.A., 2013. Nanostraw-Electroporation System for Highly Efficient Intracellular Delivery and Transfection. ACS Nano 7, 4351-4358. doi:10.1021/nn400874a 
